\title{
Revisión por pares en la Revista Médica de Chile, año 2010
}

\author{
Peer-reviewers in Revista Médica de Chile, year 2010
}

L os revisores externos de los manuscritos que se reciben en la Revista Médica de Chile cumplen una labor voluntaria, desinteresada y anónima (para los autores). La "revisión por pares" es uno de los pilares que sostienen la calidad de las revistas científicas y se ha hecho cada día más importante al aumentar la complejidad metodológica de los trabajos, en el área científica y en las especialidades clínicas. Esta colaboración demanda tiempo, esfuerzo, la revisión de bases de datos y de otras publicaciones, y la aplicación de un juicio valorativo, todo lo cual se sintetiza en un informe escrito que se pone a disposición de los editores. Es un trabajo que debe ser reconocido y valorado por los lectores de la Revista y por las instituciones en que trabajan dichos revisores. Por ese motivo, una vez al año y en el número de julio de la Revista, se identifican las personas que durante el año precedente revisaron al menos un manuscrito. Durante 2010 contamos con la participación de 569 revisores externos (7\% más que en 2009), la mayoría de los cuales revisaron dos o tres manuscritos en el año. Quienes figuran en la nómina de Miembros del Comité Editorial Asesor de la Revista, revisaron generalmente un número aún mayor.

Los informes de los revisores externos prestan una ayuda inapreciable a los autores de manuscritos, cuando expresan críticas y sugerencias que les permiten corregir omisiones o errores y, por ende, mejoran la calidad de sus manuscritos. Los editores, a su vez, se apoyan en las opiniones de los revisores externos para decidir si aceptan o rechazan un manuscrito, o solicitan a los autores que hagan cambios antes de tomar su decisión final. Todo este proceso incide directamente en la calidad de la Revista, según la aprecien sus lectores $y$ otros autores que aborden un mismo tema.

En nombre de los editores de la Revista Médica de Chile y del Directorio de la Sociedad Médica de Santiago-Sociedad Chilena de Medicina Interna, agradecemos a quienes revisaron manuscritos durante el año 2010. La nómina que se publica a continuación es un documento que acredita su participación en el proceso de "revisión por pares".

\section{Listado revisores 2010}

Patricia Abumohor G

Marisol Acuña A

Mónica Acevedo B

Mónica Acuña P

Rubén Aguayo N

Mónica Ahumada O

Cecilia Albala B

Héctor Alcaíno C

Jorge Alfaro L

Felipe Aller R

María Elena Alvarado B

Manuel Alvarez Z

Miriam Alvo A

José Amat V
Hugo Amigo C

Patricio Anabalón S

Marcela Aracena A

Timoleón Anguita C

Miguel Antúnez R

Werner Apt B

Gabriel Arancibia A

Francisco Arancibia $\mathrm{H}$

Waldo Aranda Ch

Daniel Araos $\mathrm{H}$

Teresa Aravena C

Héctor Araya I

Fernando Araya D

Magdalena Araya Q
Leopoldo Ardiles A

Leonardo Arellano H

Rodolfo Armas M

Pilar Arnaiz G

Marco Arrese J

Oscar Arteaga $\mathrm{H}$

Antonio Arteaga Ll

Eugenio Arteaga U

Sylvia Asenjo M

Carmen Paz Astete A

Eduardo Atalah S

Luis Avendaño C

Arturo Ayala B

Sylvia Armando R 
Carmen Gloria Aylwin H

Aníbal Baeza O

Fernanda Baeza G

Laura Bahamondes M

Elvira Balcells M

Eileen Blackburn T

Antonio Baquerizo M

Francisco Barriga C

María Teresa Barros D

Manuel Barros M

Adolfo Barros R

María de la Luz Bascuñán R

Gabriel Bastías S

Juan Beca I

Paula Bedregal G

Rosa Behar A

Sergio Bello S

Carlos Beltrán B

Carlos Benítez G

Zoltan Berger F

Pablo Bertín C-M

Pelayo Besa De C

Mauricio Besio R

Leandro Biagini A

Víctor Bianchi S

Lorena Binfa E

Patricia Bitar $\mathrm{H}$

Marcela Bitran C

Eileen Blackburn T

María Antonieta Blanco R

Juan Enrique Blümel M

Juan Bonbin F

María Rosa Bono M

Italo Braghetto $\mathrm{M}$

Sergio Brantes G

Sandra Braun J

Mireya Bravo L

Lucía Bronfman F

Francisca Browne L

Oscar Brunser T

Beatriz Bueno E

Daniel Bunout B

Raquel Burrows A

Marco Bustamante Z

Patricia Bustos M

María Elena Cabrera C

Jorge Calderón P

Mario Calvo A

José Miguel Campero P

María Cecilia Campos $S$

Claudia Campusano M
Mauricio Canals L

José Canessa G

Francisco Cano S

Carlos Caorsi S

María Cecilia Capetillo F

Daniel Cárcamo P

Juan Guillermo Cárcamo M

Leonardo Carmona $\mathrm{R}$

Carmen Carrasco M

Marcela Carrasco G

Luis Cartier R

César Carvajal A

Jorge Carvajal C

Bruce Cassels N.

Enrique Castellón V

Manuel Castillo N

Ramón Castillo G

Cecilia Castillo L

Marianela Castillo R

Silvia Castillo T

Daniel Castro A

Jaime Catalán G

Gabriel Cavada Ch

Iván Caviedes $\mathrm{S}$

Tulio Chacin P

Andrés Chahin F

Hernán Chamorro B

Pedro Chaná C

Víctor Charlín De G

Alejo Chávez A

Hernán Chávez G

Sara Chernilo $S$

José Chianale B

May Chomalí G

Arturo Cifuentes O

Mariana Cifuentes K

Verner Codoceo R

Miguel Concha N

Guillermo Conte L

Adela Contreras R

Luis Contreras $\mathrm{M}$

Andrea Contreras S

María Patricia Cordella M

Miguel Angel Cordero V

Aliro Correa U

Claudia Cortés M

Fanny Cortés M

Patricio Cortés P

Esteban Cortés $\mathrm{S}$

Alejandro Corvalán R

Javier Crosby R
Attila Csendes J

Miguel Cuchacovich T

Ada Cuevas M

Eduardo Cunill C

Karin D'Ottone M

Xabier De Aretxabala U

María De la Fuente $\mathrm{H}$

María Pía De la Maza C

Raúl De la Sotta T

Carlos Defilippi C

Angela Delucchi B

César Descouvieres V

Luigi Devoto C

Rodrigo Díaz G

Patricia Díaz A

Carlos Díaz C

Arturo Dell'Oro C

Violeta Díaz T

Humberto Dighero T

Javier Domínguez C

Verónica Donoso G

Archibaldo Donoso S

Alberto Dougnac L

Juan Dubernet M

Gastón Duffau T

Mauricio Duhalde N

Pilar Durruty A

Gastón Dussaillant M

Ana Dvoredsky B

Anneliese Dörr Alamos

Otto Dörr Zégers

Carlos Echeverría B

Pamela Eguiguren B

Octavio Enríquez L

Rodrigo Enríquez R

Rodrigo Erazo R

Isabel Errandonea U

Edgardo Escobar C

Pedro Escudero A

Alberto Espino E

Alejandra Espinoza E

Luis Espinoza R

Arturo Evangelista $M$

Alejandro Fajuri N

Patricia Fardella B

Rossana Faúndez H

Rosa María Feijoo $S$

Plinio Fernández B

Manuel Fernández A

Alejandra Fernández V

Wanda Fernández M 
Catterine Ferreccio R

Walter Feuerhake M

Alberto Fica C

Mauricio Fica D

Rodrigo Figueroa C

Gustavo Figueroa C

Claudia Figueroa M

Fernando Florenzano U

Ramón Florenzano U

Pedro Fodich F

Ximena Fonseca A

Patricio Fuentes G

Claudio Fullerton U

Homero Gac E

Ximena Gaete V

María Nella Gai H

Héctor Gajardo L

Jorge Gajardo N

Norbel Galanti G

Daniel Galdames P

Eugenio Galilea M

Pilar Gambra A

Patricia García C

Héctor Gatica R

Ricardo Gazitúa H

Alfredo Germain A

Juan Giaconi G

Rodrigo Gil D

Federico Gili V

Jaime Godoy F

Gonzalo Godoy J

Electra González A

Patricia Gómez M

Electra González A

Patricia González A

Sergio González B

Carlos González C

Jorge González C

Fernando González F

Jorge González H

Matías González T

Martin Gotteland

Marcos Goycoolea V

Carlos Grant Del R

Humberto Guajardo S

Rubén Guarda T

Sylvia Guardia B

Julia Guerrero P

Ernesto Guiraldes C

Miguel Gutiérrez T

María Antonieta Guzmán M
Leonardo Guzmán B

Ana María Guzmán D

Christel Hanne A

Andrés Heerlein L

Carlos Hermansen T

Antonio Hernández M

Denisse Hernández F

Guillermo Hernández G

Rodrigo Hernández $\mathrm{V}$

Patricio Herrera L

Viviana Herskovic M

Sandra Hirsch B

María Isabel Hodgson B

Juan Honeyman M

Arnold Hoppe W

Nina Horwitz C

Trinidad Hoyl M

Bessie Hunter M

Luis Ibáñez A

Fernando Ibáñez C

Humberto Ibarra $\mathrm{V}$

Gloria Icaza N

Juan Idiaquez C

Dionis Isamitt D

Fernando Ivanovic-Zuvic R

Sergio Jacobelli G

Liliana Jadue $\mathrm{H}$

Rodrigo Jaimovich $\mathrm{F}$

Patricio Jalil L

Roberto Jalil M

Alfredo Jalilie E

Lilian Jara S

Chrystal Juliet L

Carlos Juri $\mathrm{Cl}$

Mariana Kahn Ch

Elena Kakarieka W

Francisco Kamann C

Ronald Kauffmann Q

Juan Kehr S

Julieta Klaassen L

Alejandro Koppmann C

Miguel Kottow L

Jaime Labarca L

Rodolfo Lahsen M

Alexis Lama Toro

Marcela Larraguibel Q

Milton Larrondo L

Jorge Lastra $\mathrm{T}$

Pablo Lavados G

Manuel Lavados M

Federico Leighton $\mathrm{P}$
Thomas Leisewitz V

Isabel Leiva $\mathrm{R}$

David Lemus A

Luz María Letelier S

Cristian Leyton M

Claudio Liberman G

Patricia Lillo Z

Ximena Lioi $C$

Fernando Lira C

Carmen Lisboa B

Marcelo Llancaqueo V

Osvaldo Llanos L

Luis Lobos B

Vivianne Lois V

Francesca Lombardo de T

Francisco López K

Gloria López S

Néstor López V

Miriam Lorca $\mathrm{H}$

Yalda Lucero A

Pilar Macho F

Jorge Madrid A

Ana María Madrid S

Alberto Maiz G

Eduardo Maiza R

Alejandro Majlis L

Francisco Mardones S

Paula Margozzini M

Humberto Marín U

María José Martínez G

Virginia Martínez C

Claudio Martínez F

Claudio Martínez G.

María Angélica Martínez T

Loreto Massardo V

Ester Mateluna G

Marinella Mazzei P

Claudio Mella S

Sergio Mella M

Jorge Méndez S

Rubén Mercado P

Tomás Mesa L

Patricio Michaud C

Teresa Millán K

José Minguell U

Juan Miquel P

Marcelo Miranda C

Pedro Miranda C

Montserrat Molgo N

Julio Monreal U

Verónica Monsalve M 
Rodrigo Montagna M

Edson Montero C

Philippa Moore

Silvana Muñoz S

Helia Morales M

Miguel Morales S.

Rodrigo Moreno B

Gladys Moreno G

Manuel Moreno G

Raimundo Morris C

Emilio Moyano D

Leonor Moyano S

Patricia Muñoz C del V

Sara Muñoz C

Sergio Muñoz N

Santiago Muzzo B

Bettina Müller S

Oscar Neira Q

Luis Miguel Noriega $\mathrm{R}$

Rebeca Northland A

Fernando Novoa S

Danny Oksenberg R.

Luis Oliva P

Roberto Olivares C

Cristina Olivos $\mathrm{O}$

Rodulfo Oyarzún F

Alfonso Olmos C

Luis Orlandi J

Armando Ortiz P

Gonzalo Osorio A

Manuel Oyarzún G

Miguel Oyonarte G

Daniel Pacheco R

Andrés Palacios A

José Manuel Palacios J

Mario Palestini Q

Julián Panés

Karin Papapietro V

María Teresa Parada C

Manuel Parra A

Mauro Parra C

Claudio Parra R

Walter Passalacqua R

Luis Pedraza C

Alfredo Pemjean G

Blanca Peñaloza $\mathrm{H}$

Claudia Pérez A

Patricia Pérez A

Rosa María Pérez A

María Luisa Pérez Cotapos S

Gilberto Pérez P
Cecilia Perret $\mathrm{P}$

Julio Pertuzé R

Juan Pinto C

Eugenio Poch O

Juan Ignacio Pinto Q

Felipe Pollak C

Jaime Poniachik T

Lorena Porte $\mathrm{T}$

Soledad Prat M

Juan Carlos Prieto D

Alvaro Puelma P

Bárbara Puga L

Mariano Puga V

Klaus Püschel I

Katty Quezada S

Ricardo Rabagliati B

Gabriel Rada G

Fernando Radice D.

Paula Repetto L

Gabriela Repetto L

Eliana Reyes S

José Miguel Reyes V

Enrique Reynolds $\mathrm{H}$

Gloria Ribalta L

José Ribalta $\mathrm{S}$

Pablo Riedemann G

Stella Riedemann G

Horacio Ríos R

Iván Roa E

Juan Carlos Roa St

Héctor Rodríguez B

Juan Carlos Rodríguez D

Alberto Rodríguez T

Emilio Roessler B

David Rojas Z

María Graciela Rojas C

Pamela Rojas M

Alberto Rojas O

Cecilia Rojas S

Carlos Romero P

David Rojas Z

Antonio Rollán R

Leopoldo Romero N

María Inés Romero S

Pía Rosatti M

Víctor Rossel M

Eduardo Rosselot J

Emilio Roessler B

Jaime Rozowski N

Elsa Rugiero P

Manuel Ruz Ortíz
Eduardo Sabbagh P

Roque Sáenz F

Carlos Saieh A

Sofía Salas P

Fernando Saldías P

Carmen Salgado M.

Raúl Salvatici S

Karen Salvo C

Juan Eduardo Sánchez V

Alfonso Sánchez H

Hector Sánchez R

José Roberto Sánchez U

Patricio Sandoval R

Emilia Sanhueza R.

Jaime Andrés Santander T

Julia Santín M

Raimundo Santolaya C

Armando Sierralta Z

Guillermo Sanz C

Patricia Sanz C

Salvador Sarrá C

Isabel Segovia D

Ricardo Santander D

Rafael Selman A

Moisés Selman L

Rafael Sepúlveda J

Ricardo Sepúlveda M

Luis Sepúlveda M

Andrea Sepúlveda N

Irene Schiattino L

Alejandro Serani Merlo

Beatriz Shand K

Claudio Silva F

Hernán Silva I

Juana Silva O

Carlos R. Silva R

Juan Silva S

Vincenza Snow T

Pablo Soffia S

Iván Solís O

Humberto Soriano B

Diana Sosa F

Lilian Soto $S$

Verónica Soto A

Juan Ramón Soto $S$

María Angélica Sotomayor S

Marco Antonio Soza M

Eugenio Spencer O

Freddy Squella B

Laura Sterian W

Benjamín Suárez I 
György Szántho P

Patricia Tapia I

Renzo Tassara O

Andrei Tchernitchin V

Alvaro Téllez T

Eugenio Tenhamm F

Eduardo Tobar A

Paola Toche $\mathrm{P}$

Ximena Toledo C

Vinko Tomicic F

Juan Tordecilla C.

Carlos Toro A

Rafael Torres B

Marisa Torres $\mathrm{H}$

Marcelo Trucco B

Ricardo Uauy D

María Teresa Ulloa $\mathrm{F}$

Alvaro Undurraga $P$

Polentzi Uriarte G de C

Pablo Uribe G

Mario Uribe M

Christian Urzúa P

Jorge Urzúa U
Jose Rafael Valbuena M

Francisco Valdés E

Macarena Valdés C

Gloria Valdés S

Felipe Valdivia B

Gonzalo Valdivia C

Andrés Valdivieso D

Sergio Valdivieso F

María Antonieta Valenzuela P

María Teresa Valenzuela B

Eduardo Valenzuela A

Raúl Valenzuela $\mathrm{M}$

Sergio Valenzuela P

Alicia Vásquez M

Alfredo Velasco P

Nicolás Velasco F

Mauricio Venegas $S$

Patricio Ventura-J del T

Claudio Vera P-G

Renato Verdugo L

Cecilia Verdugo S

Fernando Vergara E

Marcos Vergara I
Pablo Vial C

Benjamín Vicente $\mathrm{P}$

Roque Villagra C

Eli Villalabeitia $R$

Luis Villarroel Del P

Fernando Vio del R

Ricardo von Kretschmann R

José Luis Vukasovic R

Eduardo Wainstein G

Kristina Weil P

Nelson Wohllk G

Elba Wu H

Rosita Wurgaft D

Alfredo Yáñez L

Patricio Yokota B

Ricardo Zalaquett $S$

Jaime Zamorano G

Rodrigo Zapata L

Alvaro Zúñiga D

Beatriz Zegers $\mathrm{P}$

Carlos Zehnder B

Raul Zemelman Z 